\title{
Examining the Psychological Sense of Community for Individuals with Serious Mental Illness Residing in Supported Housing Environments
}

\author{
Greg Townley $\cdot$ Bret Kloos
}

Received: 31 March 2009/Accepted: 21 July 2010/Published online: 5 August 2010

(C) Springer Science+Business Media, LLC 2010

\begin{abstract}
The psychological sense of community is an important aspect of community life; yet, it remains largely unexamined among individuals with serious mental illness (SMI). Sense of community represents the strength of bonding among community members; and this social phenomenon likely impacts the process by which individuals with SMI integrate into community life. The current study examined sense of community (SOC) for individuals with SMI by assessing the relationships between neighborhood experiences, unique factors related to SMI (e.g., mental illness diagnosis), and sense of community in the neighborhood. Participants were 402 residents of supported housing programs who used mental health services in South Carolina. Hierarchical linear regression was utilized to determine which components of community life helped to explain variability in sense of community. In total, 214 participants reported that it is very important for them to feel a sense of community in their neighborhoods. Neighbor relations, neighborhood safety, neighborhood satisfaction, neighborhood tolerance for mental illness, and housing site type emerged as significant explanatory variables of sense of community. These findings have implications for interventions aimed at enhancing SOC and community integration for individuals with SMI.
\end{abstract}

Keywords Psychiatric disability - Sense of community . Supported housing $\cdot$ Community integration .

Neighborhood

G. Townley $(\bowtie) \cdot$ B. Kloos

University of South Carolina, 1512 Pendleton Street,

Barnwell College, Columbia, SC 29208, USA

e-mail: Gregory.townley@gmail.com

\section{Introduction}

Seymour Sarason (1974) argued that the psychological sense of community constitutes the defining feature of community life. Sense of community (SOC) has been linked to increased psychological well-being (Pretty et al. 1996b; Prezza et al. 2001), perceptions of belonging and community connectedness (Sonn and Fisher 1996), and participation in the community (Chavis and Wandersman 1990). Sense of community has been investigated and argued to be relevant for diverse populations, ranging from school-age children and urban neighborhood residents to indigenous Australians and Latin American residents in Caracas (e.g., Garcia et al. 1999; McMillan and Chavis 1986; Perkins et al. 1990; Pretty et al. 1996a; Sonn 2002). However, there has been little research investigating the relationship between sense of community and well-being of persons with serious mental illness living in community settings. It may be particularly important to bolster the psychological sense of community for persons with serious mental illness because they often face societal barriers to community living, including stigma and discrimination (Cook and Jonikas 2002; Cummins and Lau 2003; Kloos 2005; Prince and Prince 2002). Similar to persons without diagnoses of mental illness, individuals with SMI function better in communities that are perceived to be tolerant and supportive (Newman et al. 1994; Wong and Solomon 2002). As mental health policy for individuals with serious mental illness (SMI) continues to shift from longterm hospitalization to community-based care, uncovering ways to enhance the psychological sense of community for this population may be a fundamental pathway by which they can move from being marginalized, undervalued members of society to becoming actively involved community members and agents of their own recovery (Nelson and Peddle 2005). 
Sense of Community Origins and Definitions

Sarason (1974) first conceptualized the psychological sense of community (PSOC) to describe the phenomena that one belongs to and is an integral part of a larger collectivity. He used the concept to represent the strength of bonding among community members and argued that it was important for personal and collective well-being. Sarason considered the absence of a psychological sense of community to be the single most disintegrating aspect of contemporary life and associated its absence with loneliness, alienation, psychological distress, and a feeling of impotence in regard to social forces. Sense of community has been found to be associated with various social and individual functioning outcomes, including length of residence, neighboring, and informal social control (Perkins et al. 1990); political participation and community involvement (Hughey et al. 1999); safety (Ziersch et al. 2005); loneliness (Pretty et al. 1996a); life satisfaction (Prezza et al. 2001); and mental health/ malaise symptoms (Ellaway et al. 2001).

Social epidemiologists have demonstrated how sense of community and other aspects of community connections play a significant role in the health, well-being, and mental health outcomes of various populations and subgroups (Pretty et al. 2006). Although sense of community has been investigated and argued to be relevant among diverse populations (e.g., Garcia et al. 1999; Perkins et al. 1990; Pretty et al. 1996a; Sonn 2002), few published studies have examined sense of community among individuals with serious mental illness despite suggestions that it is a core component that impacts their ability to successfully integrate and participate in community life (e.g., Aubry and Myner 1996; Wong and Solomon 2002; Townley and Kloos 2009).

Sense of Community and Community Integration for Individuals with Serious Mental Illness

Serious mental illness (SMI) is a term used to classify persistent psychiatric conditions that can greatly affect a person's behavior, thinking, emotions, and relationships (Kloos 2005). Diagnoses typically considered to be serious mental illnesses include schizophrenia, bipolar disorder, and severe and persistent depression. Traditionally, individuals with serious mental illness have received a high amount of intervention, often in specialized settings dedicated to the treatment of their problems. The prevailing model of such mental health care has been to take people out of community settings for care in institutional, residential treatment or hospital settings aimed at rehabilitating the patient and eventually readmitting her or him into the community (Nelson et al. 2001). According to Sarason (1974), "Few things are as destructive of the psychological sense of community....as the tendency to segregate the atypical person, to place him [sic] in a special geographic area where he will be with his 'own kind' and receive 'special handling'" (p. 161). This system of removing individuals with mental illness from their familiar communities likely increases their feelings of being rejected by society for being "different."

Fortunately, the past 30 years have seen profound changes in care for people with SMI in North America and Europe. Many of these shifts are reflected in increased, systematic focus on housing opportunities and support. Specifically, the current trend to supported housing, marked by principles of consumer choice, holding a lease for their own apartment, and flexible services, is replacing long-term institutional treatment and residential treatment facilities (Carling 1993; Nelson et al. 2001). A major distinguishing feature of the supported housing model is an emphasis on community integration, in which clients have opportunities to become citizens who are engaged in all facets of community life (Carling 1993).

As mental health care has shifted from deinstitutionalization to models that emphasize more independent community-based residence and tailored supportive care, the psychological sense of community may be particularly beneficial for numerous reasons. Sense of community may help to promote successful community integration for individuals with serious mental illness in a variety of ways, including the following: (1) increasing individuals' feelings of relatedness and interdependence with fellow community members; (2) encouraging individuals with SMI to become involved and engaged in important issues in their communities; and (3) providing individuals with a stable and dependable social structure (Aubry and Myner 1996; Cummins and Lau 2003; Townley and Kloos 2009). This, in turn, can be quite beneficial to their well-being and recovery from mental illness.

Below, we will outline components of neighborhood and community life which may impact individuals' sense of community. We chose to focus on neighborhood factors that play a role in sense of community experiences because we were interested in investigating supported housing environments and the social relationships that occur in the neighborhood. Although other factors, such as membership in community organizations and perception of influence and contribution to community life, certainly impact the sense of community experience within broader community contexts, they are not as relevant to sense of community experiences within the neighborhood. Important to note, we conceptualize 'neighborhoods' as including both as including both physical components (e.g., presence of trees, absence of structural damage) and psychosocial components (e.g., neighboring processes and perceptions of safety) (Wandersman and Nation 1998). 
Common Neighborhood Experiences that may Impact SOC for Individuals with Serious Mental Illness

\section{Neighbor Relations}

According to Unger and Wandersman (1985), neighboring involves social interaction, symbolic interaction, and the attachment of individuals with people living around them. Neighboring provides a means by which residents can establish social connections that may occur at an individual or neighborhood level (Lochner et al. 1999). In a study of 630 neighborhood residents in Italy, Prezza et al. (2001) found neighborhood relations to be more strongly associated with sense of community compared to other neighborhood and demographic variables, including years of residence, area of residence, and marital status. Frequency of neighboring behavior has also been found to explain increased sense of community and "rootedness" in the neighborhood in a study of 345 residents living in nonapartment dwellings in Winnipeg, Canada (Farrell et al. 2004). Positive neighboring experiences may be of particular importance to sense of community for individuals with SMI due to the stigma associated with mental illness.

\section{Neighborhood Safety}

Research findings suggest that greater perceptions of neighborhood safety are related to perceptions of neighborhood connection and sense of community (e.g., Zeldin and Topitzes 2002; Ziersch et al. 2005), whereas perceptions of higher neighborhood crime are negatively related to sense of community (Martinez et al. 2002). When residents perceive their neighborhoods to be unsafe, feelings of danger may overtake their daily lives, causing them to stay isolated in their homes and refuse to reach out to fellow residents (Zeldin and Topitzes 2002). This has deleterious effects on the psychological sense of community, and it appears to be particularly problematic and under-studied among individuals with serious mental illness (Yanos et al. 2004; Wong and Solomon 2002). Newman (1994) found that, among individuals with SMI living in Baltimore, Maryland, and Cincinnati and Columbus, $\mathrm{OH}$ (80\% rented apartments or homes and lived independently, whereas $20 \%$ lived rent-free with relatives or friends), concerns about being victimized by neighborhood crime appeared to overwhelm relatively positive reports about other neighborhood characteristics.

\section{Neighborhood Satisfaction}

Satisfaction with the neighborhood is a third common explanatory variable included in the sense of community literature. In a study of 1,213 residents of a transitional urban neighborhood in Nashville, TN, Chavis and Wandersman (1990) found block satisfaction and sense of community to be positively related. Similarly, Martinez et al. (2002) reported a significant relationship between satisfaction with the neighborhood and sense of community. Understanding the connection between neighborhood satisfaction and sense of community for persons with serious mental illness is of interest because data shows that when individuals with SMI are asked to rate their neighborhoods on a scale of 1 to 10, their ratings are as much as 20 percent lower than those of all other households on the block (Newman 1994). It is unclear whether this discrepancy is due to differences in perceptions or actual experiences.

\section{Unique Factors Related to SMI that may Impact SOC}

In addition to common neighborhood experiences that likely impact sense of community for all neighborhood residents, there are also unique factors that impact community life for individuals with serious mental illness.

\section{Stigma/Discrimination}

The unique experience of mental illness symptomotology can present challenges to community living for members of the SMI population. An assumption from clinical practice is that individuals with symptoms that often require intervention and staff support (e.g., persons experiencing hallucinations) could be more easily identified as having a mental illness diagnosis by neighbors. The reactions of these neighbors may include discrimination that could serve to decrease sense of community. Also, individuals with such symptoms may be less willing or less able to interact with neighbors or community members due to fears of being stigmatized, concerns that they will not be able to disguise symptoms, or because past interactions have not been positive or supportive (Cummins and Lau 2003).

In one of the only known studies to empirically assess the impact of mental illness stigma on sense of community, Prince and Prince (2002) examined the relationship between perceived stigma and community integration among 95 clients of assertive community treatment (ACT) teams. They found that clients' perceptions of stigmatization were inversely related to their sense of community. The authors suggest that further examination of the relationship between societal acceptance of mental illness and the psychological sense of community is worthy of consideration because sense of community increases the ability for individuals to function competently as community members and feel safe and satisfied with their neighborhoods. 


\section{Housing Site Type}

There is a disagreement in the literature concerning which housing situations are most conducive to sense of community ${ }^{1}$ for persons with serious mental illness. Critics of specialized, congregate living situations suggest that such housing environments promote stigmatization and segregation from broader communities (Aubry and Myner 1996)_characteristics that are antithetical to the psychological sense of community. In order for individuals with SMI to be fully integrated into the broader community and to achieve the full benefits of community living, many researchers suggest that they should live independently in neighborhoods or apartment complexes housed primarily by non-mentally ill residents (e.g., Walker and Seasons 2002; Wong et al. 2006; Wong and Solomon 2002). Living in such environments may promote more interaction with non-mentally ill neighbors and members of the broader community, thus leading to a greater sense of community (Seybolt, unpublished dissertation 2000). However, a problem with this argument is that there is evidence that living in independent apartments results in feelings of isolation and loneliness (Walker and Seasons 2002) and lower perceptions of "fitting in" (Yanos et al. 2004). It is possible that individuals with SMI residing among individuals who do not have a mental illness diagnosis are less likely to form social relationships with neighbors, and this may have destructive effects on their psychological sense of community.

\section{Rationale and Research Questions}

The overarching goal of this study was to contribute to literature and theory aimed at promoting community integration of persons with serious mental illness. An operating assumption of this study was that in order for successful and sustained community integration to occur, individuals need to experience a sense of community that supports and sustains them. Accordingly, the study aimed to provide a foundation and a rationale for future interventions aimed at enhancing sense of community in order to promote community integration for persons with serious mental illness.

This study examined the influence of explanatory variables on neighborhood sense of community for persons with SMI living in supported housing (see Fig. 1). Common neighborhood experiences (neighbor relations, neighborhood safety, and neighborhood satisfaction) and also factors that are unique to individuals with SMI (mental illness diagnosis, housing site type, and neighborhood

\footnotetext{
${ }^{1}$ Here and elsewhere we refer to sense of community with the broader community, including individuals with and without mental illness diagnoses.
}

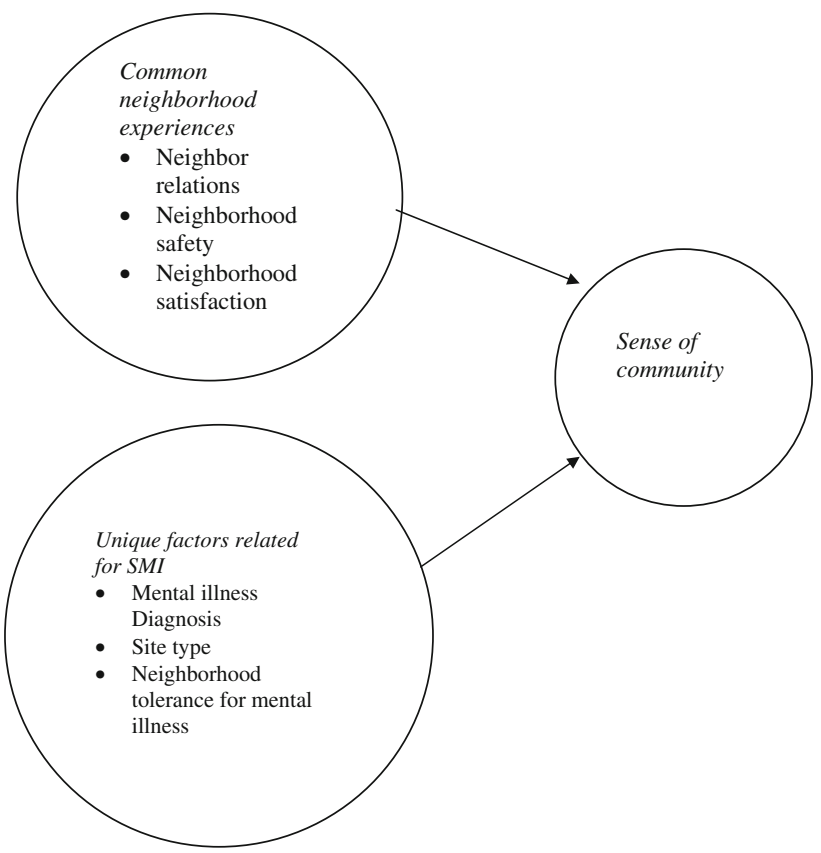

Fig. 1 Potential variables explaining variability in sense of community for persons with SMI

tolerance for mental illness) were examined. First, we hypothesized that common neighborhood experiences would explain a significant portion of variability in sense of community. Specifically, more positive relationships with neighbors and other tenants, higher reports of neighborhood safety, and higher satisfaction with the neighborhood would be related to stronger sense of community. Second, we hypothesized that unique factors related to SMI would also explain a significant portion of variability in sense of community. Specifically, participants who did not have a Psychotic Disorder, who lived in congregate-styling supported housing, and who reported higher perceptions of neighborhood tolerance for mental illness would report a stronger sense of community in their neighborhoods. Third, we hypothesized that the block of factors that were unique to people with SMI (mental illness diagnosis, housing site type, and neighborhood tolerance for mental illness) would account for additional variance in sense of community after controlling for the common neighborhood experiences (neighbor relations, neighborhood safety, and neighborhood satisfaction).

\section{Methods}

\section{Participants}

The participants in this study were 402 residents of South Carolina who had serious mental illness and lived in supported housing affiliated with the South Carolina 
Department of Mental Health (SCDMH). Each of the 17 mental health centers throughout South Carolina participated in the study and case managers at each center recruited participants. Specifically, the following systematic recruitment process was employed: (1) we developed a recruitment letter to be given to eligible participants; (2) case managers reviewed the letter $\mathrm{s}$ with eligible participants; (3) participants signed the letters if they were interested in enrolling in the study; and (4) we contacted interested participants to obtain informed consent and to complete the survey. All 763 clients who received a housing subsidy and utilized services from the SCDMH were invited to participate and a total of 533 participated in the research project. The sample for this report comes from Wave 2 of a broader study where 424 of these 533 completed both a first and a second wave of data collection between May 2005 and May 2006. The final sample size used in this report is 402; a decision was made to drop 22 of the 424 participants because they were missing more than $25 \%$ of data on the sense of community measure.

The 402 participants were nearly evenly divided by sex, with $51 \%$ of the sample being female, and the remaining $49 \%$ being male. The racial composition of the sample was as follows: $51 \%$ of the participants identified themselves as Black, $40 \%$ were White, $3 \%$ reported being bi-racial, and $4 \%$ reported other races. The average age of the participants was $46(\mathrm{SD}=10)$, with $5 \%$ being married and $50 \%$ having children, although most participant did not live with their children. The predominant diagnosis (approximately $70 \%$ ) for this sample was a Psychotic Disorder (e.g., schizophrenia), with the remaining participants having such diagnoses as Major Depression and Bipolar Disorder. Approximately $31 \%$ of the participants completed high school or obtained their GED (i.e., General Education Development, equivalent to a high school diploma) as their highest level of education, and $32 \%$ had at least some college. The vast majority of participants received Supplemental Security Income (SSI) or Social Security Disability Income (SSDI), and 17\% were working in paid employment at the time of the interview.

\section{Measures}

\section{Sense of Community}

The sense of community measure used in the current study was the Brief Sense of Community Inventory (BSCI), developed by Long and Perkins (2003). The BSCI is an eight-item scale adapted in part from the original 12-item Sense of Community Index (Perkins et al. 1990). Important to note for the current study, we asked participants to use their neighborhoods, defined by one neighborhood block in any direction, as the reference group for reporting their perceived sense of community. The BSCI was found to have enhanced psychometric properties from the original scale, and it consists of three subscales: (a) social connections, (b) mutual concern, and (c) community values. On five items, participants indicate true or false responses about sense of community in their neighborhoods; on three items, participants answer questions on a three-point Likert scale. To obtain the scale sum score and aggregate these different response options, raw-rescaling of items was used whereby the five true-false items were scored " 1 " and " 2 " and the three Likert-scale items were scored " $1,1.5$, and 2" (Long and Perkins, personal communication, 2006). The Cronbach alpha for the scale was 0.71 .

\section{Common Neighborhood Experiences}

The Housing Environment Survey (HES) scales were developed to understand the physical and social environments of supported housing for individuals with serious mental illness. Four self-report HES scales were used in the current study - the Neighbor (HES-NBR) scale, the Safety (HES-S) scale, the Residential Satisfaction (HES-RS) scale, and a subscale of the Neighborhood Social Climate (HES-NSC) scale. These scales will be discussed below. For detailed psychometric information regarding development of the HES scales, please see Kloos and Shah (2009).

The Neighbor (HES-NBR) scale includes seven items assessing neighboring behavior, including amount of contact with neighbors and material/ social support provided by neighbors. Questions are on a scale of 1 to 5 , where $1=$ "Strongly Disagree" and $5=$ "Strongly Agree." The Cronbach alpha for the scale in this dataset was 0.73 and the one-week test-retest correlation was 0.75 . To assess the validity of the Neighbor Scale, patterns of correlation were examined with potential outcomes of interest to this research. The Neighbor Scale was strongly correlated with the ISEL-12, a measure of global support $(r=.490$, $P<$.001) (Cohen and Hoberman 1983). As expected, the Neighbor Scale had a negative correlation with the UCLA Loneliness Scale $(r=-.287, P<.01)$ (Russell et al. 1980).

The Safety (HES-S) scale includes eight questions about the frequency of neighborhood safety-related occurrences, such as drug use and destruction of property. Items are on a scale of 0 to 6 , where $0=$ "Never" and $6=$ "Once a day." The Cronbach alpha for the scale in this dataset was 0.77 and the test-retest correlation was 0.79 . Tests of validity showed that the Safety Scale was negatively correlated with reports of perceived stress $(r=-.129$, $P<.05$ ) using the Perceived Stress Scale (Cohen et al. 1983) and positively correlated with reports of life satisfaction $(r=0.08, P<0.05)$ using Lehman's Quality of Life Interview (Lehman 1988). 
Neighborhood satisfaction was measuring using the sum of the following two items from the Residential Satisfaction (HES-RS) scale: "How satisfied are you with this neighborhood as a place to live?" and "How does your current neighborhood compare to your previous neighborhood?" The Cronbach alpha was 0.82. Tests of validity showed that Neighborhood Satisfaction was negatively related to perceived stress $(r=0.189, P<0.001)$ and positively related to life satisfaction $(r=0.156$, $P<0.001)$.

\section{Unique Factors Related to SMI}

The Neighborhood Social Climate (HES-NSC) scale consists of 10 questions assessing perceptions of belonging and acceptance in the neighborhood. Items are on a scale of 1 to 5 , where $1=$ "Strongly Disagree" and 5= "Strongly Agree." The Cronbach alpha for the scale in the dataset was 0.82 and the test-retest correlation was 0.67. For the current study, a 3-item subscale of the HES-NSC which assesses neighborhood tolerance and acceptance of mental illness was used. The Cronbach alpha for the subscale was 0.82. As expected, the tolerance and acceptance subscale was negatively correlated with the UCLA Loneliness Scale $(r=-0.194, P<0.001)$ and positively correlated with life satisfaction $(r=0.122, P<0.01)$.

'Site-type' is a binary dummy-coded variable collected in staff interviews that classifies individuals as living in congregate settings or non-congregate settings. The essential feature of congregate housing is that, while the sites are integrated into the broader community, they are occupied exclusively by mental health consumers. They also typically have at least a minimal level of part-time staff supervision. In contrast, non-congregate housing can range from owning a home and living by oneself to residing in a large apartment complex that may or may not house other mental health consumers. In the current study, $68 \%$ of participants lived in congregate settings and $32 \%$ lived in non-congregate setting.

Finally, the 'mental illness diagnosis' variable was taken from SC-DMH chart diagnoses. The current study classified individuals as either having a Psychotic Disoder or not having a Psychotic Disorder. The decision to handle the variable in this fashion was made because the majority of participants $(70 \%)$ had Psychotic Disorder diagnoses.

\section{Design and Procedure}

This study is part of a larger research project studying housing environments of people with serious mental illness (SMI) in order to improve housing quality, neighborhood conditions, and public perceptions of mental illness. (Wright and Kloos 2007; Townley and Kloos 2009; Kloos and Shah 2009). The majority of interviews were conducted in the participants' living space (i.e. their home or apartment), although some were completed at the local mental health center or clubhouse. Interviews were conducted with laptop computers whereby interviewers entered participants' responses into a computer program as they were given. Interviewees were compensated for their time with monetary incentives, and all data was stored confidentially on computers. The protocol was approved by the Institutional Review Boards at the University of South Carolina and the South Carolina Department of Mental Health.

\section{Data Analysis}

\section{Missing Data}

The analyses began by addressing missing data. No more than $5 \%$ of data was missing for any of the major variables under review. Multiple imputation was used to estimate missing values. This technique provides unbiased estimates of parameters and standard errors under the assumptions that data are missing at random (Cohen et al. 2003). All variables under examination, as well as any variables that could help to explain the missingness of these variables, were entered into the imputer's model. The data were then imputed ten times using SAS PROC MI, and autocorrelation plots and time-series plots were examined to ensure that the data augmentation process was successful.

Hypothesis 1 A multiple regression model was conducted to examine the ability of the common neighborhood experiences (neighbor relations, neighborhood safety, and neighborhood satisfaction) to explain significant portions of variability in sense of community. Sense of community was regressed on the block of common neighborhood experiences.

Hypothesis 2 A multiple regression model was conducted to examine the ability of the unique factors for people with SMI (mental illness diagnosis, housing site type, and neighborhood tolerance for mental illness) to explain a significant portion of the variability in sense of community. Sense of community was regressed on the block of unique factors.

Hypothesis 3 A hierarchical regression model was conducted to determine if the unique factors accounted for variance in sense of community after controlling for the common neighborhood factors. The block of common neighborhood factors was entered first, followed by the block of unique factors. The effect of including each block of explanatory variables was assessed by examining the 
increment change in $R^{2}$ and the $F$-test statistic associated with it.

It is important to note that in the original analyses, demographic covariates (race, sex, and age) were included in each regression model. However, none of these variables were significantly related to any of the other variables under investigation in the current study, and their inclusion did not significantly affect the results. Thus, the demographic covariates were removed from future analyses for the sake of parsimony and clarity.

\section{Results}

In order to provide context for the analyses which follow, we first present results from two questions asking participants to report the relative importance and experience of sense of community in their neighborhoods. When asked how important it is to feel a sense of community with people in the neighborhood, $95 \%$ of participants reported that a sense of community was important or somewhat important. Specifically, 214 participants (53\%) said that it is very important and another 169 persons (42\%) said it is somewhat important. Only 20 participants $(5.0 \%)$ said that it is not important. When asked if they actually felt a sense of community with others in their neighborhoods, 227 participants $(56 \%)$ said that they felt some sense of community, while only $128(32 \%)$ reported that they feel a strong sense of community. A minority of participants, 48 $(12 \%)$, said that they feel very little sense of community in their neighborhoods.

Hypothesis 1 The first multiple regression analysis (see Table 1) with the block of common neighborhood experiences explaining variability in sense of community was significant, $F(3,399)=44.21, P<0.001$. Each of the common neighborhood experiences (neighbor relations, neighborhood satisfaction, and neighborhood safety) was significant, and their positive beta weights revealed that they were positively related to the outcome. The model accounted for $25.7 \%$ of the variance in participants' reports of sense of community. After partialing out the effects of the other explanatory variables, neighbor relations

Table 1 Summary of multiple linear regression analysis with common neighborhood experiences accounting for variance in sense of community $(N=402)$

\begin{tabular}{lllll}
\hline Variable & $\beta$ & SE & Partial $R^{2}$ & Model $R^{2}$ \\
\hline Neighbor relations & $0.41^{* * *}$ & 0.04 & 0.13 & \\
Neighborhood satisfaction & $0.21^{* * *}$ & 0.05 & 0.05 & \\
Neighborhood safety & $0.16^{* *}$ & 0.04 & 0.04 & $0.257^{* * *}$
\end{tabular}

** $P<0.01 ; * * * P<0.001$ accounted for $13.2 \%$ of the variance in sense of community, neighborhood satisfaction accounted for $5.4 \%$ of the variance, and neighborhood safety accounted for $3.6 \%$ of the variance. Thus, the hypothesis that common neighborhoods experiences would account for a significant portion of the variability in sense of community for individuals with SMI was supported.

Hypothesis 2 The second multiple regression analysis (see Table 2) with the block of unique factors for people with SMI explaining variability in sense of community was significant, $F(3,399)=24.45, P<0.001$. The perceived neighborhood tolerance for mental illness variable was significant, and the positive beta weights revealed that it was positively related to sense of community. The housing site type variable was significantly related to sense of community, and the positive beta weight indicated that participants in congregate housing reported a higher sense of community than participants in non-congregate housing. The mental illness diagnosis variable was not significant. The model accounted for $17.2 \%$ of the variance in participants' reports of sense of community. After partialing out the effects of the other explanatory variables, neighborhood tolerance for mental illness accounted for $9.1 \%$ of the variance in sense of community, site type accounted for $6.2 \%$ of the variance, and mental illness diagnosis accounted for no additional variance. The hypothesis that unique factors for individuals with SMI would account for a significant portion of the variability in sense of community was partially supported by these analyses.

Hypothesis 3 See Table 3 for a summary of the results of the hierarchical regression testing the ability of the block of unique factors related to SMI to explain variability in sense of community after accounting for the influence of the common neighborhood factors. The common neighborhood experiences explained the bulk of the variance, $R^{2}=25.7 \%, F(3,399)=44.21, P<0.001$. The unique factors accounted for $7.20 \%$ of additional variance, and the model was significant: $F(6,396)=27.07, P<0.001$. The total model accounted for $32.9 \%$ of the variance in participants' reports of sense of community. The hypothesis that unique factors for individuals with SMI would account

Table 2 Multiple linear regression analysis with unique factors related to SMI accounting for variance in sense of community $(N=402)$

\begin{tabular}{lllll}
\hline Variable & $\beta$ & $\mathrm{SE}$ & Partial $R^{2}$ & Model $R^{2}$ \\
\hline $\begin{array}{l}\text { Neighborhood tolerance } \\
\text { for mental illness }\end{array}$ & $0.32 * * *$ & 0.04 & 0.09 & \\
Site type & $0.48^{* * *}$ & 0.06 & 0.06 & \\
Mental illness diagnosis & 0.03 & 0.06 & 0.00 & $0.172 * * *$ \\
\hline$* * * P<0.001$ & & & &
\end{tabular}


Table 3 Hierarchical linear regression analysis with common neighborhood experiences and unique factors related to SMI predicting sense of community $(N=402)$
$* P<0.05 ; * * P<0.01$ *** $P<0.001$

\begin{tabular}{llllll}
\hline Variable & $\beta$ & SE & Partial $R^{2}$ & Model $R^{2}$ & $\Delta R^{2}$ \\
\hline Step 1 (Common neighborhood experiences) & & & & \\
Neighbor relations & $0.41^{* * *}$ & 0.04 & 0.13 & \\
Neighborhood satisfaction & $0.21^{* * *}$ & 0.05 & 0.05 & \\
Neighborhood Safety & $0.16^{* *}$ & 0.04 & 0.04 & $0.257^{* * *}$ & - \\
Step 2 (Common neighborhood experiences & and unique factors) & & \\
Neighbor relations & $0.35^{* * *}$ & 0.04 & 0.11 & \\
Neighborhood satisfaction & $0.20^{* *}$ & 0.05 & 0.03 & \\
Neighborhood safety & 0.08 & 0.04 & 0.01 & \\
Site type & $0.34^{* *}$ & 0.06 & 0.06 & \\
Neighborhood tolerance for mental illness & $0.18^{*}$ & 0.04 & 0.02 & & \\
Mental illness diagnosis & 0.03 & 0.06 & 0.00 & $0.329 * *$ & $0.072^{* *}$ \\
\hline
\end{tabular}

for additional variance in sense of community after partialing out the influence of the community neighborhood experiences was supported.

\section{Discussion}

I like that we stick together here. That's important. My neighbors look out for me...It's like a family out here, people keep an eye on you. Even though I have some problems, I feel very welcome and accepted here.

-Andrea, an African American research participant living with schizophrenia

Despite facing numerous health challenges and stereotypes, persons with diagnoses of serious mental illness participating in this study expressed a desire for a sense of community and reported differing experiences of sense of community. The descriptive results from participants' responses to two questions from the Brief Sense of Community Index (BSCI) highlight the importance, although not necessarily the presence, of a sense of community in their lives. A total of 214 participants (53\% of the sample) said that it is very important for them to feel a sense of community with others in their neighborhoods. However, only $128(32 \%)$ reported actually feeling a strong sense of community. This discrepancy supports the assumption in the study that even though individuals with serious mental illness wish to feel connected to and supported by their neighborhoods, many encounter barriers that stand in the way of this actually occurring.

Experiences and Factors Associated with Sense of Community

As hypothesized, results indicate that common neighborhood experiences (neighbor relations, neighborhood safety, and neighborhood satisfaction) accounted for a significant amount of variability in sense of community. This supports common findings in the neighborhood literature (e.g., Martinez et al. 2002; Prezza et al. 2001; Zeldin and Topitzes 2002) and underscores that certain features of neighborhoods may operate similarly for persons with serious mental illness living in their own apartments as they do among people without a mental illness diagnosis. This does not mean that it is appropriate to generalize all neighborhood research findings from non-mentally ill populations to people with SMI; but it does suggest that important features that help people feel a sense of community-including safety, neighbor relations, and satisfaction-may be common across populations. Neighbor relations accounted for the largest amount of variance in sense of community, illustrating the critical role of social relationships in fostering perceptions of belonging and neighborhood connectedness.

Results also suggest that factors that are unique to persons with serious mental illness have an impact on participants' sense of community. First, perceived neighborhood tolerance for mental illness were positively associated with sense of community. This finding is important because it supports arguments that mental illness stigma has deleterious effects on sense of community (Corrigan 2005). Thus, the development of interventions aimed at increasing neighborhood tolerance for mental illness has increased importance.

Another interesting finding is that individuals living in congregate apartment housing sites (i.e., exclusively with other consumers) reported significantly higher perceptions of sense of community than individuals living in noncongregate housing sites (i.e., not exclusively with consumers in the apartment complex). It is likely that the shared experience of mental illness increases individuals' perceptions of belonging to the neighborhood and ability to contribute meaningfully to its social fabric. Although some researchers (e.g., Aubry and Myner 1996; Walker and 
Seasons 2002; Wong and Solomon 2002) have criticized congregate housing for isolating individuals with SMI and separating them from the broader community, the findings of this study actually suggest that these environments promote stronger sense of community than more "normalized" housing sites. Cummins and Lau (2003) support this idea and suggest that, as is the case with most people, individuals with disabilities may prefer to live amongst people who are similar to them and who, they believe, will contribute more strongly to perceptions of belonging and fitting in. The authors go on to argue that the goal of community integration should be to help individuals achieve a sense of community, regardless of whether this means actual integration with individuals who do not have a disability. In the words of the authors, "What is being suggested is that we stop devaluing relationships between people who have a disability, and that we lay to rest the implicit belief that associations with non-disabled people are in some sense superior" (Cummins and Lau 2003, p. 153).

Finally, the findings suggest that type of mental illness diagnosis (measured here as either having a Psychotic Disorder or not having a Psychotic Disorder) was not related to perceptions of sense of community. Diagnoses may be less important than their current functioning or acuteness of symptoms. The findings suggest that it is likely that sense of community is important for persons with mental illness irrespective of their mental illness diagnosis.

Importance of Sense of Community for Community Integration Research and Interventions

The findings from this study illustrate the importance of neighborhood and community experiences for the wellbeing of individuals with SMI. This speaks to the need for researchers and mental health centers to guide interventions aimed at promoting relationships between consumers and their neighbors, taking measures to increase neighborhood safety, determining ways to ensure consumer satisfaction with their neighborhoods, and addressing neighborhood attitudes about mental illness. The findings also illustrate the potential for congregate housing sites to promote sense of community rather than isolating and marginalizing individuals, as has often been suggested in the literature. An important caveat for interpreting these data is that it is difficult to know whether the sense of community that individuals in congregate sites reported is influenced only by fellow consumers or if it is also influenced by neighbors without a mental illness diagnosis. Future research should attempt to examine this quandary. The authors of the current study agree with Cummins and Lau (2003) in warning against assigning prescriptive solutions regarding whether social interactions and living with fellow consumers is more or less desirable than social interactions and living with non-consumers. Strategies that increase opportunities for integration and consumer choice likely will promote greater integration, sense of community, well-being.

\section{Limitations}

Several limitations need to be observed about the study. First, a cross-sectional design was employed, and the study only utilized data from one wave of interviewing. Thus causation cannot be inferred from the results. As a population study, individuals were not randomly assigned to a control or treatment group or measured pre- and posttreatment. A future study utilizing a longitudinal design could potentially track people as they move from hospital settings into the broader community, measuring potential resources and barriers that could help to explain more clearly the mechanisms through which a sense of community is developed. A potential issue with generalizeability of the findings is that they represent only the experiences of persons living in supported housing in the southeastern United States. However, given the fact that the sample was drawn from the entire state and included individuals in diverse housing sites, the findings are likely to be fairly representative.

This study relied on individuals' perceptions of neighborhood phenomena to answer the research questions. There is evidence that perceptions of environments are consistently better predictors of outcomes than objective measures, such as ratings of the physical appearance of the neighborhood, census information on crowding and SES, etc. (e.g., Stiffman et al. 1999). However, the problem with basing research findings on people's perceptions is that they are subjective-unique to the experiences of the participant and of questionable authority to be generalized to others. This methodological dilemma is compounded when participants have SMI because people are often skeptical of the accuracy of their perceptions (Newman 1995). For instance, given the nature of psychiatric symptoms, the fluctuation of symptom severity could affect participant responses; at a given time, self-reports may not be representative of the normal level of overall functioning.

\section{Future Research}

This exploratory study is the first known of its kind to systematically examine experiences and factors associated with the psychological sense of community for individuals with serious mental illness residing in supported housing 
environments. A future next step would be to employ qualitative research techniques and longitudinal studies to further understand the meaning and importance of sense of community for individuals with serious mental illness. Future studies should also examine other variables that likely impact sense of community, such as participation in community organizations and identification with various community subgroups (e.g., race, sex, and religion). Interventions could then be designed and implemented according to the specific needs and desires of individuals residing in the community. Future research can also collect information about sense of community and tolerance for mental illness from non-mentally ill neighborhood members. This information could be compared to the reports of individuals with SMI and be used in designing interventions aimed at combating stigma and increasing acceptance of mental illness within neighborhoods.

\section{Conclusions}

First and foremost, this study supports the idea that sense of community is a critical component in the community integration process for individuals with serious mental illness. Results suggest that psychosocial components of neighborhoods that are typically believed to impact sense of community among non-mentally ill persons operate similarly among individuals with SMI. However, an important addition to the literature is that certain unique aspects of community life for individuals with SMI, namely perceptions of neighborhood tolerance for mental illness and residing in a congregate housing site with fellow consumers. These factors have an impact on the sense of community over and above the influence of the common neighborhood experiences. The findings of this study suggest that understanding and promoting sense of community may be particularly important for individuals with serious mental illness due to societal stigma and problems with isolation and social exclusion.

Acknowledgments Preparation of this manuscript was supported by funding from the National Institute of Mental Health-K23MH65439. Thanks to staff and consumers of the South Carolina Department of Mental Health, who made this research possible. Thanks also to Abraham Wandersman and Shauna Cooper, Department of Psychology, University of South Carolina, for comments on an early draft of this manuscript.

\section{References}

Aubry, T., \& Myner, J. (1996). Community integration and quality of life: A comparison of persons with psychiatric disabilities in housing programs and community residents who are neighbors. Canadian Journal of Mental Health, 15(1), 5-20.
Carling, P. J. (1993). Housing and supports for persons with mental illness: Emerging approaches to research and practice. Hospital \& Community Psychiatry, 44(5), 439-449.

Chavis, D. M., \& Wandersman, A. (1990). Sense of community in the urban environment: A catalyst for participation and community development. American Journal of Community Psychology, 18, $55-81$.

Cohen, J., Cohen, P., West, S. G., \& Aiken, L. S. (2003). Applied multiple regression/ correlation analysis for the behavioral sciences (3rd ed.). Mahwah, NJ: Lawrence Erlbaum Associates.

Cohen, S., \& Hoberman, H. M. (1983). Positive events and social supports as buffers of life change stress. Journal of Applied Social Psychology, 13, 99-125.

Cohen, S., Kamarck, T., \& Mermelstein, R. (1983). A global measure of perceived stress. Journal of Health and Social Behavior, 24, 385-396.

Cook, J. A., \& Jonikas, J. A. (2002). Self-Determination among mental health consumers/ survivors: Using lessons from the past to guide the future. Journal of Disability Policy Studies, 13(2), 87-95.

Corrigan, P. (Ed.). (2005). On the stigma of mental illness: Practical strategies for research and social change. Washington, DC: American Psychological Association.

Cummins, R. A., \& Lau, A. L. D. (2003). Community integration or community exposure? A review and discussion in relation to people with an intellectual disability. Journal of Applied Research in Intellectual Disabilities, 16, 145-157.

Ellaway, A., Macintyre, S., \& Kearns, A. (2001). Perceptions of place and health in socially contrasting neighborhoods. Urban Studies, 38(12), 2219-2316.

Farrell, S., Aubry, T., \& Coulombe, D. (2004). Neighborhoods and neighbors: Do they contribute to personal well-being? Journal of Community Psychology, 32(1), 9-25.

Garcia, I., Giuliani, F., \& Wiesenfeld, E. (1999). Community and sense of community: The case of an urban barrio in Caracas. Journal of Community Psychology, 27, 727-740.

Hughey, J., Speer, P. W., \& Peterson, N. A. (1999). Sense of community in community organizations: Structure and evidence of validity. Journal of Community Psychology, 27, 97-113.

Kloos, B. (2005). Creating new possibilities for promoting liberation, well-being, and recovery: Learning from experiences of psychiatric consumers/ survivors. In G. Nelson \& I. Prillitensky (Eds.), Community psychology: In pursuit of well-being and liberation (pp. 426-447). London: MacMillan.

Kloos, B., \& Shah, S. (2009). A social ecological approach to investigating relationships between housing and adaptive functioning for persons with serious mental illness. American Journal of Community Psychology, 44(3-4), 316-326.

Lehman, A. F. (1988). The well-being of chronic mental patients: Assessing their quality of life. Archives of General Psychiatry, 40, 369-373.

Lochner, K., Kawachi, I., \& Kennedy, B. P. (1999). Social capital: A guide to its measurement. Health and Place, 5, 259-270.

Long, D. A., \& Perkins, D. D. (2003). Confirmatory factor analysis of the sense of community index: Development of a brief SCI. Journal of Community Psychology, 31(3), 279-296.

Martinez, M. L., Black, M., \& Starr, R. H. (2002). Factorial structure of the perceived neighborhood scale (PNS): A test of longitudinal invariance. Journal of Community Psychology, 30(1), 23-43.

McMillan, D. W., \& Chavis, D. M. (1986). Sense of community: A definition and theory. Journal of Community Psychology, 14, 6-23.

Nelson, G., Lord, J., \& Ochocka, J. (2001). Empowerment and mental health in community: Narratives of psychiatric consumer/survivors. Journal of Community and Applied Social Psychology, 11, $125-142$. 
Nelson, G., \& Peddle, S. (2005). Housing and support for people who have experienced serious mental illness: Value base and research evidence. Waterloo, ON: Wilfrid Laurier University. Unpublished manuscript.

Newman, S. J. (1994). The housing and neighborhood conditions of persons with severe mental illness. Hospital \& Community Psychiatry, 45(4), 338-343.

Newman, S. J. (1995). The accuracy of reports on housing and neighborhood conditions by persons with severe mental illness. Psychosocial Rehabilitation Journal, 18(3), 129.

Newman, S. J., Rechovsky, J. D., Kaneda, K., \& Hendrick, A. M. (1994). The effects of independent living on persons with chronic mental illness: An assessment of the Section 8 Certificate program. The Milbank Quarterly, 72(1), 171-198.

Perkins, D., Florin, P., Rich, R., Wandersman, A., \& Chavis, D. (1990). Participation and the social and physical environment of residential blocks: Crime and community context. American Journal of Community Psychology, 18, 83-115.

Pretty, G. H., Andrews, L., \& Collett, C. (1996a). Exploring adolescents' sense of community and its relationship to loneliness. Journal of community psychology, 22, 346-357.

Pretty, G., Bishop, B., Fisher, A., \& Sonn, C. (2006). Psychology sense of community and its relevance to well-being and everyday life in Australia. The Australian Psychology Society.

Pretty, G. H., Conroy, C., Dugay, J., Fowler, K., \& Williams, D. (1996b). Sense of community and its relevance to adolescents of all ages. Journal of Community Psychology, 24(4), 365-379.

Prezza, M., Arrici, M., Roberti, T., \& Tedeschi, G. (2001). Sense of community referred to the whole town: Its relations with neighboring, loneliness, life satisfaction, and area of residence. Journal of Community Psychology, 29(1), 29-52.

Prince, P. N., \& Prince, C. R. (2002). Perceived stigma and community integration among clients of assertive community treatment. Psychiatric Rehabilitation Journal, 25(4), 323-331.

Russell, D., Peplau, L. A., \& Cutrona, C. E. (1980). The revised UCLA loneliness scale: Concurrent and discriminant validity evidence. Journal of Personality and Social Psychology, 39, $472-480$.

Sarason, S. B. (1974). The psychological sense of community: Prospects for a community psychology. San Francisco: JosseyBass.

Seybolt, D. C. (2000). Residential factors predicting community integration and quality of life for persons with serious mental illness. South Carolina: University of South Carolina. Unpublished manuscript.

Sonn, C. C. (2002). Immigrant adaptation: Exploring the process through sense of community. In A. T. Fisher, C. C. Sonn, \& B.
J. Bishop (Eds.), Psychological sense of community: Research, applications, and implications (pp. 205-222). New York, NY: Kluwer Academic/ Plenum Publishers.

Sonn, C. C., \& Fisher, A. T. (1996). Psychological sense of community in a politically constructed group. Journal of Community Psychology, 24, 417-430.

Stiffman, A. R., Hadley-Ives, E., Elze, D., Johnson, S., \& Dore, P. (1999). Impact of environment on adolescent mental health and behavior: Structural equation modeling. American Journal of Orthopsychiatry, 69(1), 73-86.

Townley, G., \& Kloos, B. (2009). Development of a measure of sense of community for individuals with serious mental illness residing in community settings. Journal of Community Psychology, 37(3), 363-380.

Unger, D. G., \& Wandersman, A. (1985). The importance of neighbors: The social, cognitive, and affective component of neighboring. American Journal of Community Psychology, 13, $139-169$

Walker, R., \& Seasons, M. (2002). Supported housing for people with serious mental illness: Resident perspectives on housing. Canadian Journal of community Mental Health, 21(1), 137-151.

Wandersman, A., \& Nation, M. (1998). Urban neighborhoods and mental health: Psychological contributions to understanding toxicity, resilience, and intervention. American Psychologist, 53(6), 647-656.

Wong, Y.-L. I., Metzendorf, D., \& Min, S.-Y. (2006). Community integration: Perspectives from mental health consumers and providers. Social Work in Mental Health, 4(3), 45-59.

Wong, Y.-L. I., \& Solomon, P. (2002). Community integration of persons with psychiatric disabilities in supportive independent housing: Conceptual model and methodological issues. Mental Health Services Research, 4(1), 13-28.

Wright, P. A., \& Kloos, B. (2007). Housing environment and mental health outcomes: A levels of analysis perspective. Journal of Environmental Psychology, 27(1), 79-89.

Yanos, P. T., Barrow, S. M., \& Tsemberis, S. (2004). Community integration in the early phase of housing among homeless persons diagnosis with severe mental illness: Successes and challenges. Community Mental Health Journal, 40(2), 133-150.

Zeldin, S., \& Topitzes, D. (2002). Neighborhood experiences, community connection, and positive beliefs about adolescents among urban adults and youth. Journal of Community Psychology, 30(6), 647-669.

Ziersch, A., Baum, F. E., MacDougall, C., \& Putland, C. (2005). Neighborhood life and social capital: The implications for health. Social Science and Medicine, 60, 71-86. 